\title{
Characterization of Self-Assembled Rosette Nanotubes using SEM, TEM, AFM and XRD
}

Jae-Young Cho, Grigory Tikhomirov, Mounir El-Bakkari and Hicham Fenniri

National Institute for Nanotechnology, National Research Council (NINT-NRC) and Department of Chemistry, University of Alberta, 11421 Saskatchewan Drive, Edmonton, Alberta T6G 2M9, Canada

As the importance of characterization for nanotechnology has increased in the past few years, more research has been carried out on soft nanostructured materials using scanning electron microscopy (SEM), transmission electron microscopy (TEM), atomic force microscopy (AFM) and $x$-ray diffraction (XRD) [1-3]. However, the effect of experimental conditions on data acquisition by SEM, TEM, AFM and XRD is not fully understood. In this paper, the critical factors that strongly affect quality of the structural information are presented for a soft self-assembled organic material Rosette Nanotubes (RNTs) (Fig. 1)

For the SEM and TEM characterization of RNTs, the importance of sample preparation and conditions of electron microscopy (EM) will be presented. For the AFM characterization, three different substrates: mica, highly ordered pyrolytic graphite (HOPG) and thin amorphous carbon grid were used for the understanding of interaction between RNT and substrate surface. Through XRD characterization of RNT, additional information about periodic features of the structure over larger length scales will be presented (Fig. 2). Optimal sample preparation, deposition technique (spin coating and drop casting) and characterization conditions will be presented and interpretation of data will be presented [4].

References

[1] Rahul Chhabra, Jesus G. Moralez, Jose Raez, Takeshi Yamazaki, Jae-Young Cho, Andrew J. Myles, Andriy Kovalenko, and Hicham Fenniri, J. Am. Chem. Soc. 2010, 132, 32-33.

[2] Ross S. Johnson, Takeshi Yamazaki, Andriy Kovalenko, and Hicham Fenniri, J. Am. Chem. Soc. 2007, 129, 5735-5743.

[3] Pascal Jonkheijm, Atsushi Miura, Magdalena Zdanowska, Freek J. M. Hoeben, Steven De Feyter, Albertus P.H. J. Schenning, Frans C. De Schryver, and E.W. Meijer, Angew. Chem. Int. Ed. 2004, 43, 74-78.

[4] This research program was supported by the National Institute for Nanotechnology and the National Research Council CANADA. 

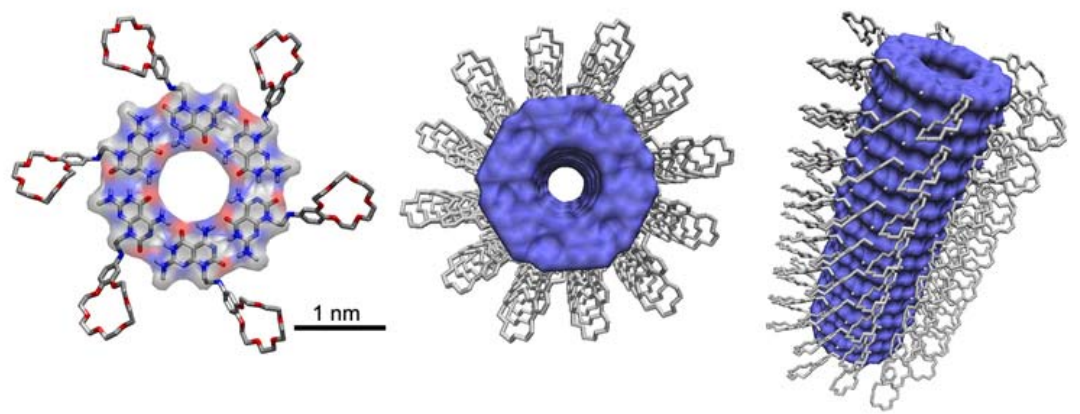

FIG. 1. Rosette structure obtained from the self-assembly of $6 \mathrm{G}^{\wedge} \mathrm{C}$ motifs (left) and top (middle) and side (left) views of the rosette nanotubes.
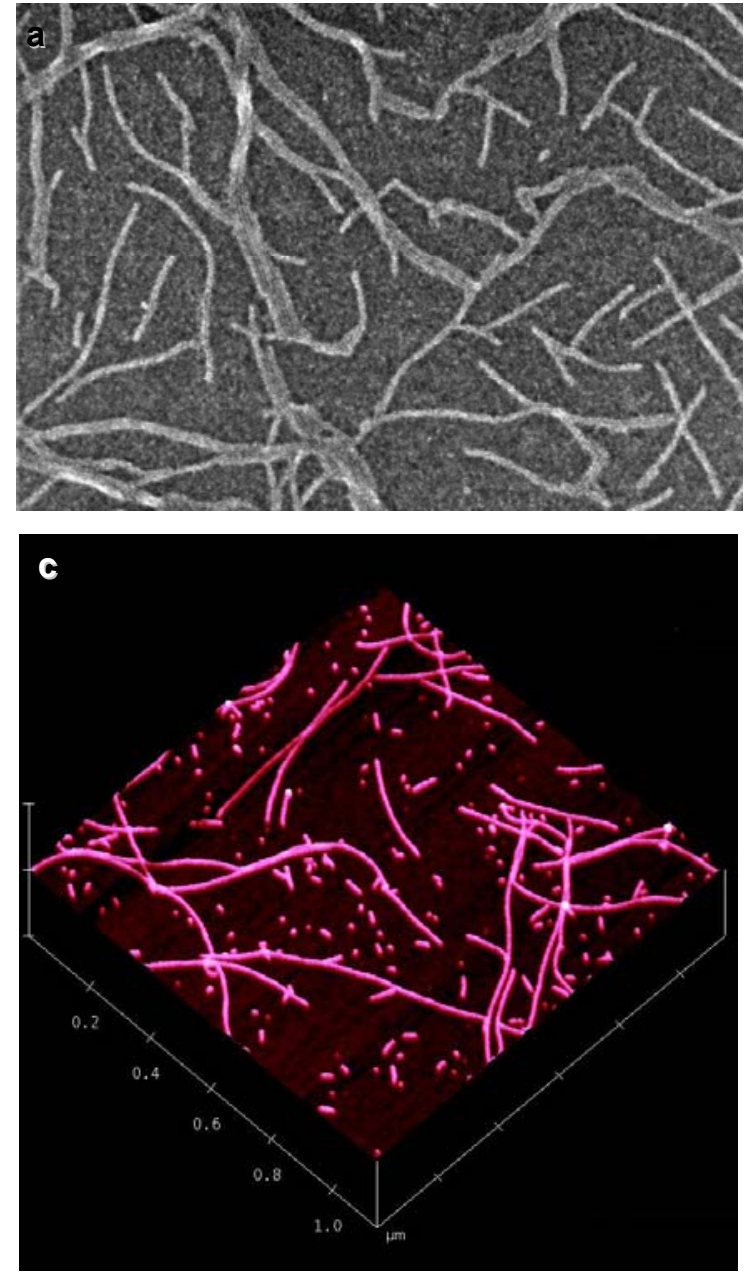
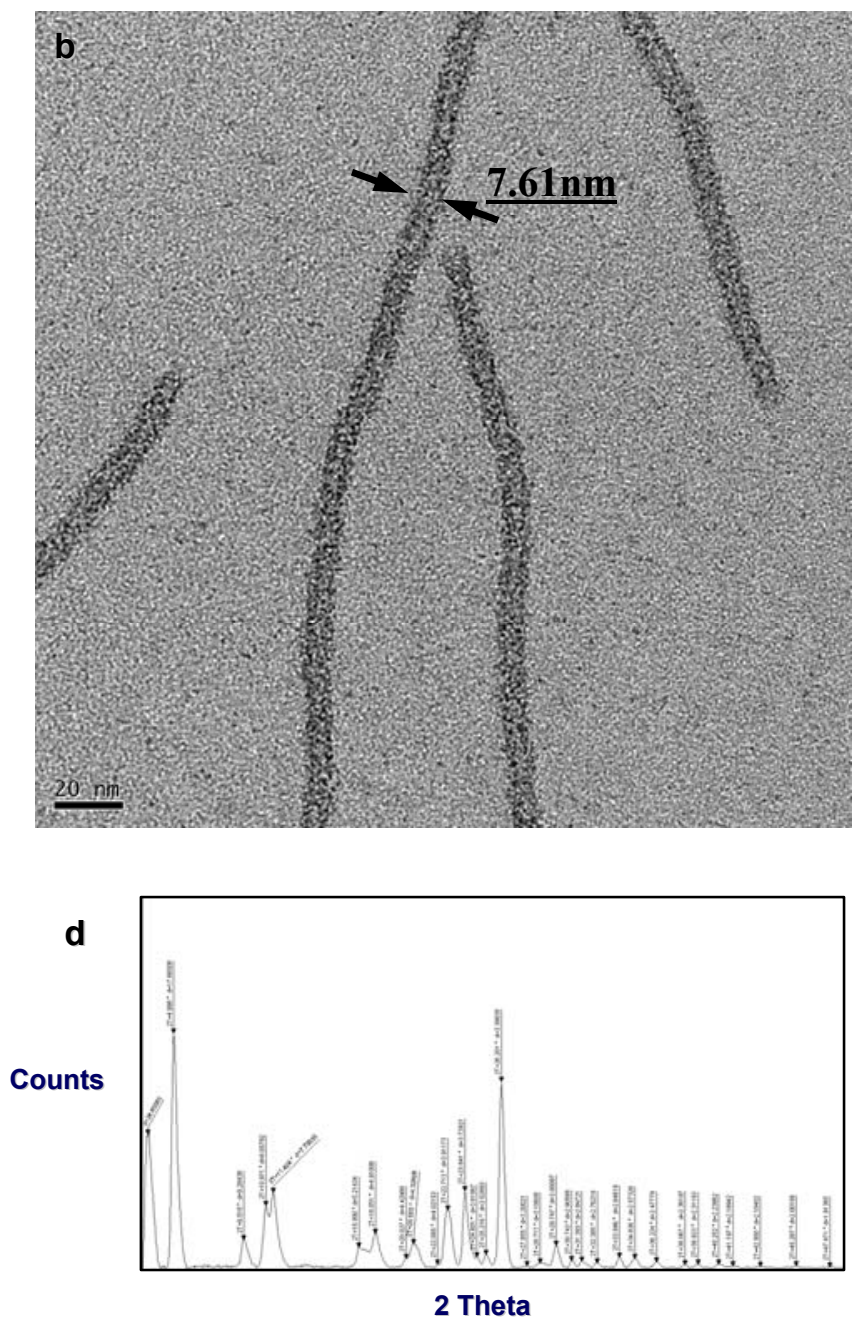

FIG. 2. Characterization of RNTs using (a) SEM, (b) TEM, (c) AFM and (d) XRD. 